\title{
ECHINOCOCCUS GRANULOSUS HAPLOTYPES IN THE SCIENTIFIC LITERATURE
}

\section{Dear Editor}

Echinococcus granulosus ( $\mathrm{Eg}$ ) is a parasite causing an incidental infection in humans. The disease can be observed as a hydatid cyst in the liver and lungs and is found in dogs and wolves as definitive hosts. It is considered a neglected infectious disease which has rarely been studied in Mexico ${ }^{1}$. In several studies worldwide, the genetic study of mitochondrial DNA (mtDNA) sequencing of cox 1 and nad1 partial sequences is predominant. However, studies of haplotypes obtained or concatenated (analysis combining sequences of two or more genes) are scarce.

The research group in Medical Science of the Universidad de La Frontera, located in the Araucania Region in Chile where $\mathrm{Eg}$ is endemic, recently created a study group to analyze this disease from the clinical and basic sciences viewpoint. Consequently, during different studies performed, the group has identified the various haplotypes informed in the literature and is interested in contributing and sharing with the scientific community, the information collected and classified according to the main characteristics of the disease.

\section{REFERENCES}

1. Totomoch-Serra A, Manterola C. Importance of genotyping cystic echinococcosis in humans from Mexico. Arch Med Res. 2020;51:63-4.
Eg has high genetic variability and the classification applied in different studies is diverse. In reference to the different populations informed, the analysis of haplotypes can help to trace changes in the evolutive forces, to perform in phylogeography analysis and to determine the global genetic structure ${ }^{2,3}$. By having the information available, the researchers performing mtDNA haplotype analysis can easily access published data to compare and design their haplotype analysis. The information may be accessed as supplementary material (Supplementary Table 1).

\section{SUPPLEMENTARY DATA}

Supplementary data are available at Revista de Investigación Clínica online (www.clinicalandtranslationalinvestigation.com). These data are provided by the corresponding author and published online for the benefit of the reader. The contents of supplementary data are the sole responsibility of the authors.

2. Ito A, Yanagida T, Nakao M. Recent advances and perspectives in molecular epidemiology of Taenia solium cysticercosis. Infect Genet Evol. 2016;40:357-67

3. Spotin A, Boufana B, Ahmadpour E, Casulli A, Mahami-Oskoue $\mathrm{M}$, Rouhani S, Assessment of the global pattern of genetic diversity in Echinococcus multilocularis inferred by mitochondrial DNA sequences. Vet Parasitol. 2018;262:30-41.

\section{Armando Totomoch-Serra ${ }^{1,2 *}$, Nayely García-MÉndez ${ }^{1}$, And Carlos Manterola Ma $^{1,3,4}$ \\ ${ }^{1} \mathrm{PhD}$ Program in Medical Sciences, Universidad de La Frontera, Temuco, Chile; ${ }^{2}$ Department of Genetics and Molecular Biology, Center for Research and Advanced Studies, Instituto Politécnico Nacional, Mexico City, Mexico; ${ }^{3}$ Center for Excellence in Morphological and Surgical Studies (CEMyQ), Universidad de La Frontera, Temuco, Chile; ${ }^{4}$ Department of Surgery, Universidad de La Frontera, Temuco, Chile}

*Corresponding author:

Armando Totomoch-Serra

E-mail: totomoch@gmail.com
Received for publication: 29-04-2020

Approved for publication: 07-05-2020

DOI: $10.24875 / R I C .20000194$

0034-8376 / (c) 2020 Revista de Investigación Clínica. Published by Permanyer. This is an open access article under the CC BY-NC-ND license (http://creativecommons.org/licenses/by-nc-nd/4.0/). 